\title{
Lokal fortgeschrittenes Plattenepithelkarzinom der Mundhöhle: Präoperative Induktion?
}

\author{
Die Langzeitergebnisse nach \\ Resektion und Rekonstruktion \\ lokal fortgeschrittener, operabler \\ oraler Plattenepithelkarzinome \\ sind trotz nachfolgender Strah- \\ len- oder Radiochemotherapie \\ mit 5-Jahres-Überlebensraten von \\ 50-60\% unbefriedigend. Ist eine \\ präoperative Chemotherapie \\ sinnvoll?
}

$\mathrm{n}$ der offenen randomisierten PhaseIII-Studie erhielten die Patienten im Prüfarm eine Induktionstherapie mit zwei Zyklen TPF (Docetaxel $75 \mathrm{mg} / \mathrm{m}^{2}$ an Tag 1, Cisplatin $75 \mathrm{mg} / \mathrm{m}^{2}$ an Tag 1 , 5-Fluorouracil $750 \mathrm{mg} / \mathrm{m}^{2}$ an den Tagen 1-5 als Dauerinfusion). Es folgten die radikale Tumorresektion mit vollständiger „neck dissection“ und eine adäquate Rekonstruktion. Nach 4-6 Wochen wurde in konformaler Standardtechnik oder intensitätsmoduliert mit 54-66 Gy über sechs Wochen bestrahlt. Im Kontrollarm wurde auf die präoperative Chemo- therapie verzichtet. Primäres Studienziel war das Überleben, sekundäre Endpunkte die lokale Kontrolle und die Sicherheit der Therapie.

Von 256 randomisierten Patienten mit lokal fortgeschrittenem Plattenepithelkarzinom der Mundhöhle in den Stadien III und IVA erhielten 222 die vollständige Studientherapie. Etwa $70 \%$ waren Männer, der Anteil der Nichtraucher war mit $51 \%$ überraschend hoch und die Zahl der Patienten mit regelmäßigem Alkoholkonsum mit $41 \%$ überraschend niedrig.

Die präoperative TPF-Therapie war mit einer Remissionsrate von 80,6\% (Komplettremission 8,1\%; partielle Remission 72,6\%) hocheffektiv. Nach einer medianen Nachbeobachtungszeit von 30 Monaten konnte aber weder im Gesamtüberleben (OS) noch im krankheitsfreien Überleben (DFS) ein Gewinn durch die präoperative TPF-Chemotherapie nachgewiesen werden (Hazard Ratio 0,977 bzw. 0,974; p =0,918 bzw. $0,897)$. Nach zwei Jahren überlebten insgesamt $68,8 \%$ der Patienten im experi- mentellen und 68,2\% im Kontrollarm, auch das krankheitsfreie Überleben nach zwei Jahren unterschied sich mit 62,2\% bzw. 63,6\% nicht.

In der Analyse der zahlreichen Subgruppen hatten nur Patienten mit gutem klinischem und/oder pathologischem Ansprechen ( $\leq 10 \%$ vitale Tumorzellen) ein besseres Überleben und eine bessere lokoregionäre und FernmetastasenKontrolle.

Fazit: Insgesamt, so die Autoren, konnte die TPF-Induktionstherapie das Überleben der Patienten mit fortgeschrittenem Plattenepithelkarzinom der Mundhöhle nicht verbessern. Die Autoren selbst fordern weitere Studien, speziell um diejenigen Patienten zu erkennen, die besonders von einer präoperativen Chemotherapie profitieren könnten. Außerdem müsste diese sicher über die zwei präoperativen Zyklen hinaus ausgedehnt werden.

Brigitte Schalhorn

Zhong LP et al. Randomized phase III trial of induction chemotherapy with docetaxel, cisplatin, and fluorouracil followed by surgery versus upfront surgery in locally advanced resectable oral squamous cell carcinoma. J Clin Oncol. 2013;31(6):744-51.

\section{Hypopharynxkarzinom: Induktionschemotherapie statt Laryngektomie?}

\section{Lange Zeit musste bei größeren Karzinomen des Hypopharynx der Kehlkopf obligat mit entfernt werden. Die aktuellen 10-Jahres-Daten der EORTC-Studie 24891 zeigen: eine Induktionschemotherapie mit anschließender Bestrahlung führt zu vergleichbaren Ergebnissen wie die konventionelle Behandlung.}

n die randomisierte Studie waren 202 Patienten mit Plattenepithelkarzinomen des Hypopharynx oder des lateralen Epipharynx eingeschlossen. Bei allen lag ein Tumor in den Stadien T2-T4, N0-2b ohne Fernmetastasen vor. In der ersten Studiengruppe erfolgte eine totale Laryngektomie mit partieller Hypopharyngektomie und „neck dissection“ sowie nachfolgender Radiotherapie (50 Gy fraktioniert, zusätzlich $14 \mathrm{~Gy}$ als Booster bei positiven Schnitträndern oder Lymphknoten). Patienten der Induktionsgruppe dagegen erhielten eine initiale Chemotherapie mit bis zu drei Zyklen (100 mg Cisplatin/m² an Tag 1, 1.000 mg/ $\mathrm{m}^{2}$ 5-Fluorouracil an den Tagen 1-5). Bei komplettem makroskopischem Ansprechen in der Endoskopie schloss sich eine Radiotherapie (50 Gy fraktioniert, zusätzlich 20 Gy als Booster in der Tumorregion) an. Ergab sich auch nach dem dritten Zyklus kein komplettes Ansprechen, wurden die Patienten konventionell operiert (34 von 97 Patienten).

Nach median 10,5 Jahren war bei 54 Patienten nach konventioneller Therapie und bei 49 nach Induktionschemotherapie eine Progression der Erkrankung aufgetreten. Die 10-Jahres-Raten für progressionsfreies Überleben betrugen $8,5 \%$ bzw. 10,8\%. Im gleichen Beobach- tungszeitraum verstarben 81 Patienten in der Gruppe mit konventioneller sowie 83 im Studienarm der Induktionschemotherapie. Dies entsprach einem prozentualen 10-Jahres-Gesamtüberleben von 13,8 vs. $13,1 \%$.

Fazit: Nach den 10-Jahres-Daten dieser Studie kann man bei Hypopharynxkarzinomen durch eine Induktionschemotherapie in einigen Fällen eine Laryngektomie vermeiden. Weder progressionsfreies noch Gesamtüberleben waren dadurch beeinträchtigt. Das ändert allerdings nichts an der insgesamt schlechten Prognose der Patienten. Andreas Fischer

Lefebvre JL et al. Laryngeal preservation with induction chemotherapy for hypopharyngeal squamous cell carcinoma: 10 -year results of EORTC trial 24891. Ann Oncol. 2012;23(10):270814. 